\title{
Analytical solutions for exchange bias and coercivity in ferromagnetic/antiferromagnetic bilayers
}

\author{
J. Geshev \\ Instituto de Física - UFRGS, Caixa Postal 15051, 91501-970, Porto Alegre, RS, Brazil
}

(Received 27 January 2000; revised manuscript received 11 April 2000)

\begin{abstract}
Analytical expressions have been derived for the exchange bias field, coercivity, and effective anisotropy field in ferromagnetic/antiferromagnetic bilayers in the framework of a model assuming the formation of a planar domain wall at the antiferromagnetic side of the interface with the reversal of the ferromagnetic orientation. It is shown that there are five different sets of analytical expressions for the hysteresis loop displacement and coercivity, which depend on the interfacial exchange coupling strength and ferromagnetic anisotropy, and only one expression for the effective anisotropy field. These expressions are compared with the previously reported theoretical results, and the validity of the latter is discussed. It is shown that in the framework of the present model, the hysteresis loop, ac susceptibility, and ferromagnetic resonance measurements of exchange anisotropy should give the same values for the exchange bias field. The difference between the exchange bias field values, estimated experimentally by ac susceptibility and through hysteresis loop measurements for $\mathrm{Co} / \mathrm{CoO}$ bilayers, is explained as well.
\end{abstract}

\section{INTRODUCTION}

The exchanged anisotropy, ${ }^{1}$ one of the manifestations of which is a shift in the magnetization curve away from the zero-field axis, refers to the exchange interactions at the interface between ferromagnetic (FM) and antiferromagnetic (AF) materials. Exchange bias in thin films has found important technological application in such devices as magnetoresistive sensors. ${ }^{2,3}$ Even though this phenomenon was discovered four and a half decades ago and despite extensive investigations and technological importance, its microscopic origin has not yet been understood.

The simplest theory, ${ }^{1}$ assuming that the exchange bias effect arises from the exchange coupling at an uncompensated FM/AF interface, leads to an exchange field which is typically two orders of magnitude larger than the experimentally observed ones. To explain this discrepancy, two alternative models which do not require an uncompensated FM/AF interface, a random-field model by Malozemoff, ${ }^{4}$ and a planar domain-wall model by Mauri and co-workers, ${ }^{5}$ have been proposed. The latter model suggests the formation of a domain wall at the interface with the reversal of the FM orientation, which would effectively lower the interfacial energy cost of reversing the FM layer without removing the condition of strong interfacial FM/AF coupling. Unlike the model of Mauri et al., the AF domain walls in the Malozemoff's model are normal to the interface, and the exchange bias is interpreted in terms of random exchange fields due to interface roughness. However, this model has intrinsic features which depend on details of microstructure.

The spin-flop model proposed recently by $\mathrm{Koon}^{6}$ suggested the existence of unidirectional anisotropy in thin films with a fully compensated AF interface. The micromagnetic numerical calculations indicate the stability of the interfacial exchange coupling with a perpendicular orientation between FM and AF axes directions. One limitation of Koon's model is that he restricted the motion of the spins during field reversal to the plane parallel to the interface. Schulthess and Butler, ${ }^{7}$ based on a microscopic Heisenberg model, yielded interfacial spin-flopped state similar to Koon's. However, allowing the spins to have out-of-plane components, they had contrary conclusions with respect to exchange biasing: their calculations indicated enhanced uniaxial anisotropy or enhanced coercivity, but not a shifted loop.

The above-cited models, as well as others proposed in the literature, have attained different degrees of agreement with existing experimental results. Models which, like the one of Mauri et al., include the existence of AF domain wall in exchange coupled systems, account quantitatively for the $10^{-2}$ reduction of the exchange field from the ideal interface model case, ${ }^{4,5,8-10}$ as well as for accumulative memory effects of the thermal and field history of real FM/AF bilayers. ${ }^{11}$ Up to now, however, there exists no basic, generally applicable, predictive theory or model, ${ }^{12,13}$ the reason being the strong dependence of the exchange bias on the particular chemical and spin structure at the FM/AF interface for each real system.

Anisotropic magnetoresistance ${ }^{8}$ and ac susceptibility measurements ${ }^{14}$ found an exchange anisotropy energy several times larger than that obtained from hysteresis loop measurements. Two other reversible measurement techniques, ferromagnetic resonance (FMR) and Brillouin light scattering, gave values about $20 \%$ less and $25 \%$ larger than those measured via superconducting quantum interference device (SQUID) magnetometry and magnetoresistance, respectively. ${ }^{15,16}$ This fact has led some authors to classify the measuring techniques in two categories, reversible and irreversible, interpreted with different models., ${ }^{9,12}$ Very recently, Xi et al., ${ }^{17}$ based on the model of Mauri et al., investigated theoretically the irreversible and reversible measurements of exchange anisotropy and derived expressions relating the exchange field measured by various techniques with the interface coupling field and the effective domainwall field.

In Mauri et al.'s work ${ }^{5}$ magnetization curves have been calculated numerically on the basis of the coherent rotation model for various interfacial exchange coupling constants and fixed FM anisotropy, and the main characteristics 
of easy axis (coercivity and loop displacement) and hard axis (effective anisotropy field) curves have been plotted as a function of the interface exchange parameter $\lambda$. We tried to reproduce the above dependencies using numerical calculations, and found some quantitative discrepancies between our results and those of Mauri et al. ${ }^{5}$ We also succeeded to derive analytical expressions for the normalized coercivity $h_{c}$, the loop shift $h_{e b}$, and the effective anisotropy field $h_{a}$. Excellent agreement between our analytical and numerical data has been found.

The purpose of the present work was to find analytical expressions for $h_{c}, h_{e b}$, and $h_{a}$ as functions of $\lambda$. The particular case of the value of FM anisotropy constant $K_{F M}$ considered in the original work of Mauri et al. has been treated as well. The validity of the analytical expressions previously derived is also discussed.

\section{MODEL}

The model proposed by Mauri et al. ${ }^{5}$ assumes that the AF layer with uniaxial anisotropy is infinitely thick and the domain wall can form at the AF side of the interface. At a certain distance from the interface, a FM film with thickness $t$ much smaller than the thickness of the domain wall follows. The spins of the ferromagnet rotate coherently, all including the same angle $\beta$ with the FM easy magnetization axis, which is chosen to coincide with the AF one. Let $A$ and $K$ be the exchange stiffness and crystalline anisotropy constants in the antiferromagnet, respectively, and $\alpha$ the angle of the AF moment at the interface with respect to the easy axis. When the magnetic field $H$ is applied along the easy direction, the total magnetic energy in units of $\sigma_{w}(=2 \sqrt{A K}$, which is the energy per unit surface of a $90^{\circ}$ domain wall in the $\mathrm{AF}$ ), can be written as

$$
\delta=-\cos \alpha-\lambda \cos (\alpha-\beta)+\mu \cos ^{2} \beta-h \cos \beta,
$$

where the constant terms are neglected. The first term corresponds to the domain-wall energy, the second term is the exchange energy with $\lambda=J_{E} / \sigma_{w}$ the normalized exchange, where $J_{E}$ is the effective interfacial coupling energy. The third term is the anisotropy energy on the ferromagnet with $\mu=-K_{F M} t / \sigma_{w}$ the normalized anisotropy constant with positive $K_{F M}$, and the last term is the magnetostatic energy with $h=H M_{s} t / \sigma_{w}$ the normalized magnetic field, where $M_{s}$ is the FM saturation magnetization.

For the limiting cases $\lambda \ll 1$ and $\lambda \gg 1$, one can readily obtain $^{5}$ the exchange bias field $H_{e b}$

$$
H_{e b}= \begin{cases}-J_{E} / M_{s} t & \text { for } \lambda \ll 1 \\ -\sigma_{w} / M_{s} t & \text { for } \lambda \gg 1 .\end{cases}
$$

More details for the applicability of this approach can be obtained analytically, or by numerically calculating magnetization curves of the ferromagnetic film from Eq. (1) by finding the angles $\alpha_{0}$ and $\beta_{0}$ for which $\delta$ is at minimum. For the numerical calculations, we used two-variable energy minimization procedure similar to the one used in our previous works. ${ }^{18,19}$ In the hysteresis loop calculations, the step for the normalized field was $10^{-3}$, the initial step for the

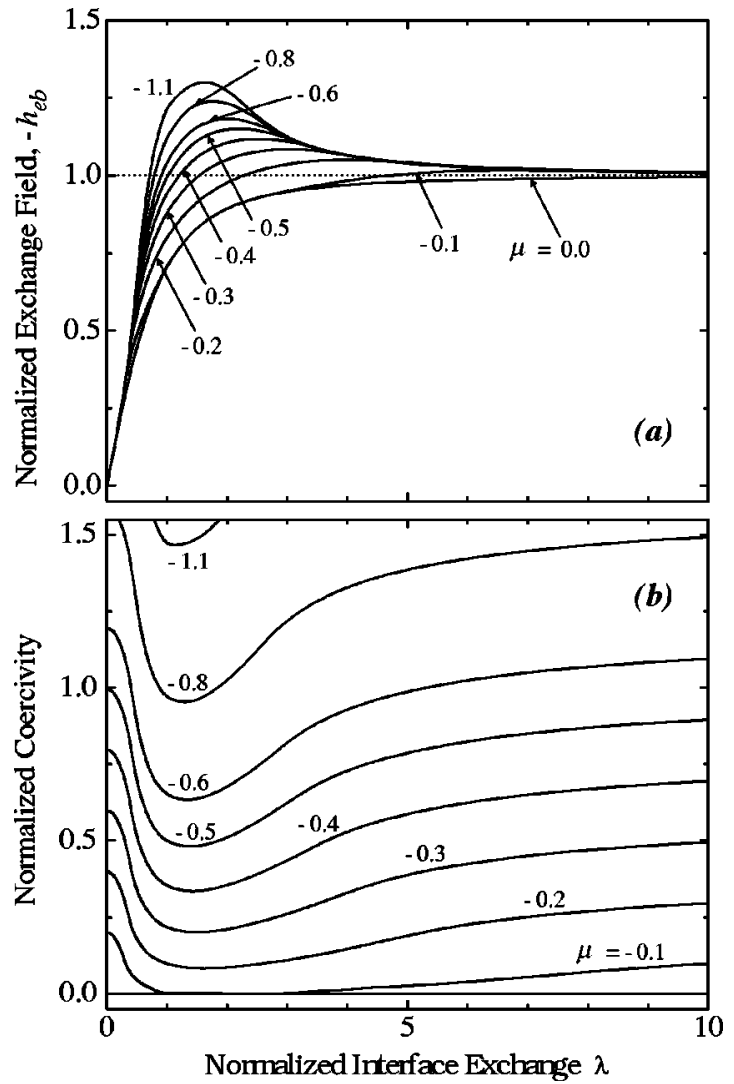

FIG. 1 . The normalized loop shift $-h_{e b}$ (a) and coercivity $h_{c}$ (b) as a function of the exchange parameter $\lambda$ for several normalized ferromagnetic anisotropy constant $\mu$ values.

angles $\alpha$ and $\beta$ was $10^{-3}$ rad, and the angles corresponding to the energy minimum were determined to an accuracy of $10^{-16} \mathrm{rad}$.

Representative $h_{c}(\lambda)$ and $h_{e b}(\lambda)$ dependencies, obtained from the numerically calculated easy axis magnetization curves, are plotted in Fig. 1 for several $\mu$ values. All $h_{c}$ versus $\lambda$ curves show minima, which become relatively deeper and sharper with the increase of the uniaxial FM anisotropy. It can be seen that for $\mu$ values smaller than a certain critical value $\mu_{\text {crit }}$, there are no $\lambda$ regions with zero coercivity. The exchange bias field curves present maxima which, like the minima in the $h_{c}(\lambda)$ dependencies, become more pronounced for higher FM anisotropy and shift to the range of lower $\lambda$ values. Note that for weak and strong interfacial coupling, all the $h_{e b}(\lambda)$ curves coincide.

In Fig. 2, the symbols denote the normalized fields $h_{c}$, $h_{e b}$, and $h_{a}$ versus $\lambda$ for the case of $\mu=-0.25$, obtained from the numerically calculated magnetization curves; $h_{a}$ is the effective anisotropy field $\left(=M_{s} / \chi\right)$, where $\chi$ is the initial susceptibility, obtained from the hard axis magnetization curves. A comparison between these curves and the ones given by Mauri et al. ${ }^{5}$ (Fig. 3 in their paper) shows that there are significant quantitative differences between these two sets of curves. The solid lines in Fig. 2 are the corresponding analytical solutions. There is an excellent agreement between our analytical results and the numerically obtained data.

In what follows, it is demonstrated how these analytical solutions have been derived. The analytical results are com- 


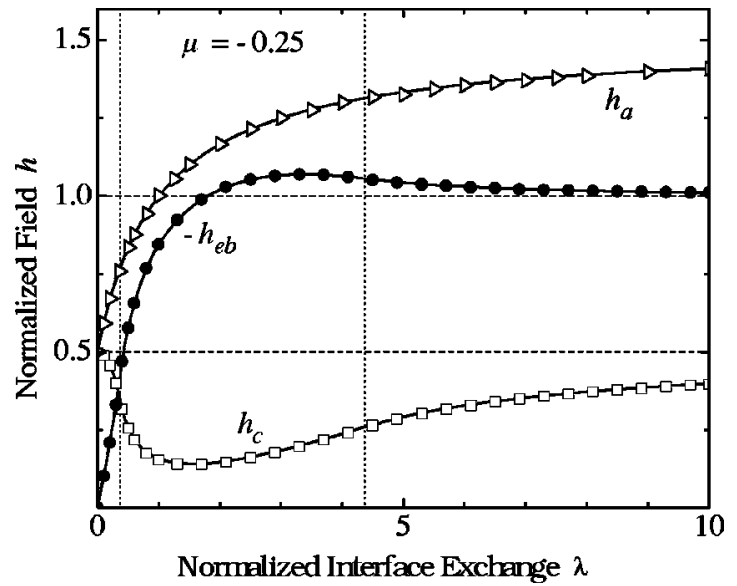

FIG. 2. The normalized coercivity $h_{c}$, the loop shift $-h_{e b}$, and the effective anisotropy field, $h_{a}=M_{s} / \chi$ versus the exchange parameter $\lambda$ for a fixed ferromagnetic anisotropy constant $\mu$ $=-0.25$. The symbols represent the data calculated numerically. The solid lines are the corresponding analytical solutions. The dotted vertical lines separate the regions, for which different analytical expressions have been used.

pared with the numerical and theoretical ones previously reported, and the validity of the latter has been discussed.

The total normalized magnetic energy is given by Eq. (1). Only positive $\lambda$ and negative $\mu$ (uniaxial FM anisotropy) have been considered. The equilibrium state is determined by equating the partial derivatives to $\alpha$ and $\beta$ to zero:

$$
\frac{\partial \delta}{\partial \alpha}=\sin \alpha+\lambda \sin (\alpha-\beta)=0
$$

and

$$
\frac{\partial \delta}{\partial \beta}=-\lambda \sin (\alpha-\beta)-\mu \sin 2 \beta+h \sin \beta=0 .
$$

For fixed $\mu$, this gives a relation between $\alpha, \beta$ and $h$ by eliminating of $\lambda$

$$
\sin \alpha=\mu \sin 2 \beta-h \sin \beta .
$$

The stability of this equilibrium is then examined with the aid of the conditions

$$
\frac{\partial^{2} \delta}{\partial \alpha^{2}} \frac{\partial^{2} \delta}{\partial \beta^{2}}-\left(\frac{\partial^{2} \delta}{\partial \alpha \partial \beta}\right)^{2}>0
$$

and

$$
\frac{\partial^{2} \delta}{\partial \alpha^{2}}>0
$$

or

$$
\frac{\partial^{2} \delta}{\partial \beta^{2}}>0
$$

In these conditions, $\alpha$ is eliminated by using Eq. (4).

\section{ANALYTICAL RESULTS}

There are two possible situations for a magnetization curve to intercept the abscissa: (i) $\beta=\pi / 2$ for a certain field value $h_{\alpha, \pi / 2}$ during a continuous magnetization rotation, and (ii) the magnetization vector jumps from a state with $\beta$ $<\pi / 2$ to a state with $\beta>\pi / 2$ when the stability of the equilibrium at the former state is lost. Following the procedure described in the previous section, the field $h_{\alpha, \pi / 2}$ is easy to be obtained. If $\lambda$ is in the range determined by the condition $B_{1} \geqslant 1$ [obtained with the aid of Eqs. (5)-(7)], where

$$
B_{1}=\lambda^{2}\left(1+\lambda^{2}\right)^{-3 / 2}(-2 \mu)^{-1},
$$

then

$$
h_{\alpha, \pi / 2}=-\frac{\lambda}{\sqrt{1+\lambda^{2}}} .
$$

The condition $B_{1} \geqslant 1$ gives the $\lambda$ range for fixed $\mu$ for which $\cos \beta=0$. There is a limiting $\mu$ value for this to happen, which can be determined by isolating $\mu$ from the expression $B_{1}=1$ and calculating its first and second derivatives to $\lambda^{2}$. From the conditions $\partial \mu / \partial \lambda^{2}=0$ and $\partial^{2} \mu / \partial\left(\lambda^{2}\right)^{2}<0$, one obtains that $\cos \beta$ can be zero if $\mu \in\left(-3^{-3 / 2}, 0\right)$.

For $\mu \leqslant-3^{-3 / 2}$, the point of intersection of the magnetization curve with the abscissa is determined as follows. At given $h$ the equilibrium loses its stability at certain values of $\beta$ depending on $h$ [the expression in Eq. (5) shifts from positive to negative, whereas the other expression used, Eqs. (6) or (7) remains positive]. Substituting this critical value pair of $h$ and $\beta$ in Eq. (3) then gives a relation between $\lambda, \mu$, and the critical value of $h_{\alpha, \beta}$ at which the equilibrium is lost. The critical fields thus obtained are

$$
h_{0,0}=-\frac{\lambda}{1+\lambda}+2 \mu,
$$

$$
\begin{gathered}
h_{0, \pi}=-\frac{\lambda}{1-\lambda}-2 \mu, \\
h_{\pi, \pi}=\frac{\lambda}{1-\lambda}-2 \mu,
\end{gathered}
$$

and, for all other cases

$$
\begin{aligned}
h_{\alpha, \beta}= & \frac{\mu}{16 \lambda\left(\lambda^{2}-c^{2}\right)}\left[12\left(\lambda^{6}+1\right)+72 c\left(\lambda^{4}+1\right)\right. \\
& \left.+\left(68-3 / \mu^{2}\right) \lambda^{4}+112 c^{2}\left(\lambda^{2}+1\right)+(240 c+68) \lambda^{2}\right],
\end{aligned}
$$

where

$$
c=\frac{1}{2}\left[\lambda^{4 / 3}(-2 \mu)^{-2 / 3}-\lambda^{2}-1\right] .
$$

The conditions determining which of the expressions (9)(13) for the critical fields must be used, are examined with the aid of Eqs. (5)-(7) for each particular case.

Let $h_{1}$ and $h_{2}$ be the normalized fields for which the descending and ascending parts of a hysteresis loop intercept the abscissa, respectively. Using these notations, the normalized coercivity and exchange bias field are

$$
h_{c}=-\frac{h_{1}-h_{2}}{2},
$$




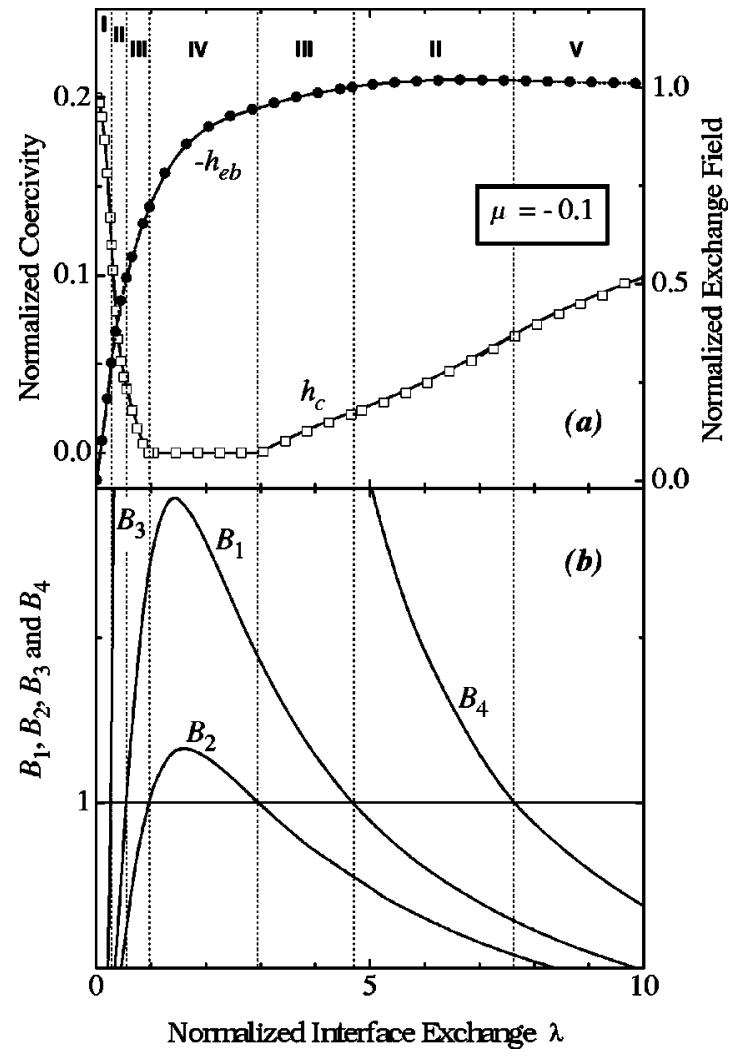

FIG. 3. (a) The normalized coercivity $h_{c}$ and the loop shift $-h_{e b}$ as a function of the exchange parameter $\lambda$ for ferromagnetic anisotropy constant $\mu=-0.1$. The symbols denote the numerical calculation data, and the solid curves correspond to the analytical solutions. (b) Dependencies of the expressions $B_{1}, B_{2}, B_{3}$, and $B_{4}$ on $\lambda$, which determine the boundaries of the five regions in (a).

$$
h_{e b}=\frac{h_{1}+h_{2}}{2} .
$$

The dependencies $h_{c}(\lambda)$ and $h_{e b}(\lambda)$ for the representative case of $\mu=-0.1$ are shown in Fig. 3(a). The symbols are the results obtained from the numerical calculations, and the solid curves are the corresponding analytical solutions. Below, the expressions for $h_{c}$ and $h_{e b}$ for both cases, $\mu$ $\leqslant-3^{-3 / 2}$, and $\mu \in\left(-3^{-3 / 2}, 0\right)$, are given.

\section{Expressions for $h_{c}(\lambda)$ and $h_{e b}(\lambda)$ for $\mu \in\left(-3^{-3 / 2}, 0\right)$}

The $h_{c}(\lambda)$ and $h_{e b}(\lambda)$ dependencies shown in Fig. 3(a) are for $\mu=-0.1$ which is larger than $-3^{-3 / 2}$, so they can be used as a demonstration for the present case. The $\lambda$ range is divided into five regions, from $\mathrm{I}$ to $\mathrm{V}$, for which different expressions for $h_{c}$ and $h_{e b}$ have been derived. Some representative hysteresis loops are plotted in Fig. 4 for eight different $\lambda$ values, corresponding to the regions indicated in Fig. 3. It is most convenient to begin the description from region IV, where $h_{c}=0$.

\section{Region IV}

The coercivity is zero because in this region $h_{\alpha, \pi / 2}$ $\leqslant h_{0,0}$. In the descending part of the loop, the magnetization starts to deviate from saturation at $h_{0,0}$. The corresponding magnetization drop, however, is not big enough for a state

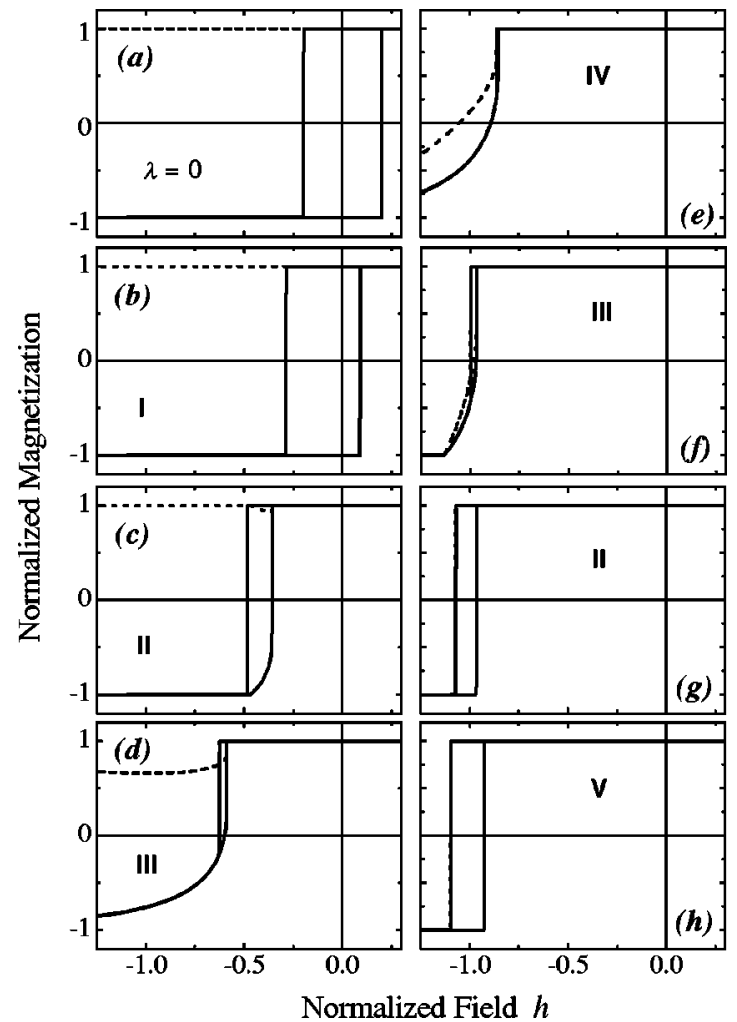

FIG. 4. Easy axis hysteresis loops for $\mu=-0.1$ and $\lambda=0.15$ (b), 0.40 (c), 0.75 (d), 2.0 (e), 4.0 (f), 7.0 (g), and 9.0 (h), which correspond to regions I-IV, III, II, and V in Fig. 3, respectively. The loop for $\lambda=0$ is given in (a) as well. The solid lines represent the normalized to $M_{s}$ magnetization of the ferromagnetic layer; the dashed lines represent the normalized magnetization of the interface antiferromagnetic plane, when different from the ferromagnetic one.

with $\beta>\pi / 2$ to be reached, and the curve crosses the abscissa by continuous rotation at $h=h_{\alpha, \pi / 2}$. The left and right boundaries for $\lambda$ for this region can be determined by equating the difference $h_{\alpha, \pi / 2}-h_{0,0}$ to zero, which gives a condition $B_{2}=1$, where

$$
B_{2}=\lambda(2 \mu)^{-1}\left[(1+\lambda)^{-1}-\left(1+\lambda^{2}\right)^{-1 / 2}\right] .
$$

If $\lambda$ fulfills the condition $B_{2}>1$, then $h_{\alpha, \pi / 2}<h_{0,0}$, the fields $h_{1}$ and $h_{2}$ are both equal to $h_{\alpha, \pi / 2}$ [Eq. (9)], thus

$$
h_{c}=0 \text {, }
$$

$$
h_{e b}=h_{\alpha, \pi / 2}=-\frac{\lambda}{\sqrt{1+\lambda^{2}}} .
$$

It is then clear that the limiting FM anisotropy value for zero coercivity in the $h_{c}(\lambda)$ curves is the above obtained limiting value for which $\cos \beta=0$, i.e., $\mu_{\text {crit }}=-3^{-3 / 2}$ $(\sim-0.19245)$. Note that in this region, $h_{e b}$ does not depend explicitly on $\mu$ and, subsequently, all the $h_{e b}(\lambda)$ curves with $\mu \in\left(-3^{-3 / 2}, 0\right)$ will coincide. This can be seen in Fig. 1(a): the curve for $\mu=-0.1$ coincides with the one for $\mu=0.0$ for $\lambda \in(0.97,2.95)$.

An example for a magnetization curve for this region is shown in Fig. 4(e) for $\lambda=2.0$. The curve does not show hysteresis, it is only shifted from the origin by $h_{\alpha, \pi / 2}$. 


\section{Region III}

This is the $\lambda$ range for which the hysteresis loop intercepts the zero magnetization axis by an irreversible jump in the descending part and by continuous rotation in the ascending one. The condition for this is $B_{1}>1$, excluding the region for which $B_{2} \geqslant 1$ (i.e., region IV). Here $h_{1}=h_{0,0}, h_{2}$ $=h_{\alpha, \pi / 2}$, and

$$
\begin{gathered}
h_{c}=\frac{\lambda}{2}\left(\frac{1}{1+\lambda}-\frac{1}{\sqrt{\left(1+\lambda^{2}\right)}}\right)-\mu, \\
h_{e b}=-\frac{\lambda}{2}\left(\frac{1}{1+\lambda}+\frac{1}{\sqrt{\left(1+\lambda^{2}\right)}}\right)+\mu .
\end{gathered}
$$

The hysteresis loops for $\lambda=0.75$ and 4.0, plotted in Figs. 4(d) and (f), correspond to the left and right parts of this region, respectively.

\section{Region II}

Here, the left boundary for $\lambda$ values, obtained using Eqs. (5) - (7), is determined by the condition $B_{3}>1$ (and consequently $\lambda<1$ ), where

$$
B_{3}=\lambda^{2}(2 \mu)^{-1}(\lambda-1)^{-3},
$$

and the right one from $B_{4}>1$ (and as a result $\lambda>1$ ), where

$$
B_{4}=-B_{3},
$$

excluding the regions III and IV. In this case, $h_{1}=h_{0,0}[\mathrm{Eq}$. (10)], $h_{2}=h_{\alpha, \beta}$ [Eq. (13)], and $h_{c}(\lambda)$ and $h_{e b}(\lambda)$ are obtained using Eqs. (15) and (16). The hysteresis loops for this region shown in Figs. 4(c) and (g), are for $\lambda=0.4$ and 7.0, respectively. The loops intercept the abscissa by irreversible jumps in both parts, but there is still reversible rotation for a certain $h$ range for the ascending part of the loops.

The dependencies of $B_{1}, B_{2}, B_{3}$, and $B_{4}$ on $\lambda$, which determine the boundaries of the five regions, are shown in Fig. 3(b).

\section{Region I}

This is the small $\lambda$ range: $\lambda$ varies between 0 and a $\lambda$ value determined by the conditions $\lambda<1$ [which comes from Eq. (6) $]$ and $B_{3}=1$, i.e., the left boundary of region III. In this region $h_{1}=h_{0,0}, h_{2}=h_{0, \pi}$, and

$$
\begin{gathered}
h_{c}=-\frac{\lambda^{2}}{1-\lambda^{2}}-2 \mu, \\
h_{e b}=-\frac{\lambda}{1-\lambda^{2}} .
\end{gathered}
$$

The limiting case of $\lambda \ll 1$ implies $h_{e b}=-\lambda$, the result obtained by Mauri et al. A loop corresponding to this region, with $\lambda=0.15$, is given in Fig. 4(b).

\section{Region V}

This is the last region ( $\lambda$ higher than a certain value), where the conditions obtained with the aid of Eqs. (5) and (6) are $B_{4} \leqslant 1$ and $\lambda>1$. The critical fields are $h_{1}$ $=h_{0,0}, h_{2}=h_{\pi, \pi}$, and

$$
\begin{gathered}
h_{c}=\frac{\lambda}{1-\lambda^{2}}-2 \mu, \\
h_{e b}=\frac{\lambda^{2}}{1-\lambda^{2}} .
\end{gathered}
$$

Using Eq. (25), one derives the result of Mauri et al. for this limiting case of $\lambda \gg 1: h_{e b}=-1$. The hysteresis loop shown in Fig. 4(h) for $\lambda=9.0$, is an example for this high $\lambda$ values region.

\section{Expressions for $h_{c}(\lambda)$ and $h_{e b}(\lambda)$ for $\mu \leqslant-3^{-3 / 2}$}

In Fig. 2, where the coercivity, exchange bias field, and the effective anisotropy field versus $\lambda$ dependencies for $\mu$ $=-0.25$ are shown, the $\lambda$ range is divided into three regions only. Due to the sufficiently small $\mu$ value in this case, there is no $\lambda$ for which the magnetization curve intercepts the abscissa by continuous rotation and therefore there are no $\lambda$ regions corresponding to regions III and IV in Fig. 3(a). The analytical expressions for $h_{c}(\lambda)$ and $h_{e b}(\lambda)$ for the rest three regions are the same as those derived above for the case of $\mu \in\left(-3^{-3 / 2}, 0\right)$.

\section{Effective anisotropy field $\boldsymbol{h}_{\boldsymbol{a}}$}

The effective anisotropy field is obtained numerically from the hard axis magnetization curves [the last term in Eq. (1) should be replaced by $h \cos (\pi / 2-\beta)]$ as $h_{a}=M_{s} / \chi$. The analytical expression for $h_{a}$ is easily derived following, e.g., the procedure used by $\mathrm{Xi}$ et al.: ${ }^{17}$ in the small $h$ range, the angles $\alpha$ and $\beta$ are very small, $\sin \beta \approx \beta$, and direct relationship between $\beta$ and $h$ is obtained by eliminating $\alpha$ from Eq. (2). The result is $\beta=-h \sin (\pi / 2) /[\lambda /(1+\lambda)-2 \mu]$. The initial susceptibility is then $\chi=\partial\left[M_{s} \cos (\pi / 2-\beta)\right] / \partial h$ for $h$ $=0$, which gives

$$
\chi=M_{s} /\left(\frac{\lambda}{1+\lambda}-2 \mu\right) \quad \text { and } \quad h_{a}=M_{s} / \chi=\frac{\lambda}{1+\lambda}-2 \mu \text {. }
$$

In terms of $J_{E}, \sigma_{w}$, and $K_{F M}$, the above expression for $h_{a}$ gives the normalized to $\sigma_{w}$ denominator in the expression for $\chi$ in the work of $\mathrm{Xi}$ et al., ${ }^{17}$ as expected. It can be seen that $h_{a}=-h_{0,0}$ for all $\mu$.

\section{DISCUSSION}

Equations (23) and (25) show that the exchange bias field does not depend explicitly on the uniaxial anisotropy for $\lambda$ in the regions $\mathrm{I}$ and $\mathrm{V}$. This explains the coincidence of all $h_{e b}(\lambda)$ curves in these regions. With the decrease of the FM anisotropy, the $\lambda$ values for which $h_{e b}$ start to depend on $\mu$, 
increase for weak interfacial coupling and decrease for strong coupling. It is interesting to note that

$$
h_{c}= \begin{cases}h_{e b} \lambda-2 \mu & \text { in region } \mathrm{I} \\ h_{e b} / \lambda-2 \mu & \text { in region } \mathrm{V} .\end{cases}
$$

As it was mentioned above, there are some quantitative discrepancies between the results for $\mu=-0.25$ shown in Fig. 2, and those of Mauri et al. ${ }^{5}$ plotted in Fig. 3 in their work for the same FM anisotropy value. Probably, the rather large initial step of $3^{\circ}$ for $\alpha$ and $\beta$ in the energy minimization used there is responsible for the deviations of their curves from the analytically obtained ones. Some of the conclusions drawn in their paper should be corrected in the light of the analytical solutions derived here.

The steady-state situation (loop shifted from the origin by $\sim 1$ ) is virtually reached for $\lambda$ close to 10 and not to 4 . The coercivity and the effective anisotropy field, however, are far from saturation even for rather high $\lambda$ values. The analytical expression for $h_{c}(\lambda)$ for this case is given by Eq. (24) and even for $\lambda=40$ the coercivity $h_{c}(40)=0.95 h_{c}(\infty)$. The condition $B_{4} \leqslant 1$ determines that the hysteresis loops for $\mu$ $=-0.25$ become square for $\lambda$ higher than 4.365 and not for $\lambda \gg 1$. The same remark is valid for a value of $\lambda$ above which the reversal of the FM magnetization is accompanied by a total reversal of the interface AF magnetization. Another point to be corrected is the one stating that for $\mu=-0.25$ the most important result of the easy axis calculation is that the exchange field can only become smaller than 1 when $\lambda$ is smaller than 1: the actual value is $\sim 1.8$.

Very recently, $\mathrm{Xi}$ et al. ${ }^{17}$ on the basis of the model of Mauri et al. derived analytical expressions for the exchange bias field considering three different irreversible and reversible measurement techniques. For the case of easy axis hysteresis loop measurements, they investigated the simple case of zero FM anisotropy, and the analytical expression obtained for the absolute value of the normalized to $\sigma_{w}$ exchange bias field, $J_{h l}$ (i.e., $J_{h l} / \sigma_{w}=\left|h_{e b}^{h l}\right|$ ), is

$$
\frac{J_{h l}}{\sigma_{w}}=\frac{J_{E}}{\sqrt{J_{E}^{2}+\sigma_{w}^{2}}},
$$

It is easy to demonstrate that in terms of $\lambda$ this expression equals $\lambda / \sqrt{1+\lambda^{2}}$. The same expression for the absolute value of the loop displacement for region IV comes from Eq. (19). It can be applied for region IV only, i.e., for $\lambda$ for which $B_{2} \geqslant 1$; however, when $\mu=0$, it is valid for all $\lambda$. The corresponding to this case $h_{e b}(\lambda)$ curve is also shown in Fig. 1 .

Expressions for ac susceptibility measurements, $J_{\mathrm{ac}}(\lambda)$ for all $\lambda$, and for FMR measurements, $J_{\mathrm{FMR}}(\lambda)$ for weak and strong interfacial coupling, have also been obtained by $\mathrm{Xi}$ et al. ${ }^{17}$ Their $J_{\mathrm{FMR}}(\lambda)$ expressions coincide with the ones derived here for regions $\mathrm{I}$ and $\mathrm{V}$, respectively. This means that hysteresis loop and FMR measurements of exchange anisotropy for magnetic systems whose behavior can be described in the framework of the Mauri et al.'s model, should not give different values for the exchange coupling for these two limiting regions. This conclusion is in excellent agreement with the results of Rubinstein et al., ${ }^{20}$ which investigated the ferromagnetic resonance field shift in a $\mathrm{CoO} /$

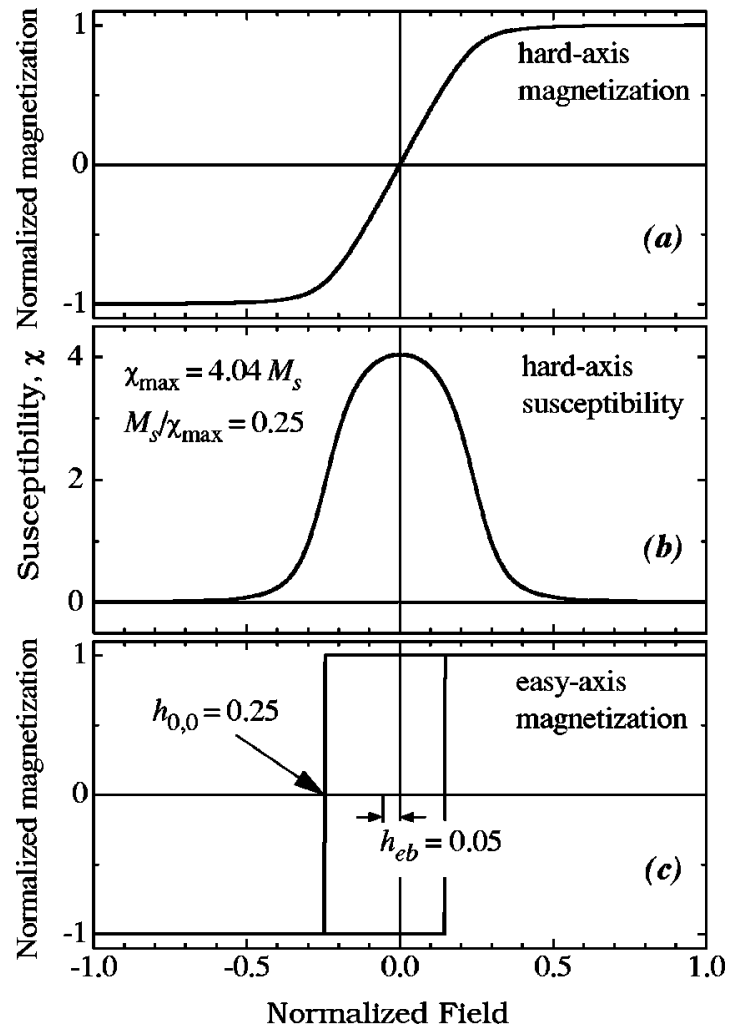

FIG. 5. (a) Hard axis magnetization curve, (b) the corresponding susceptibility, and (c) the easy axis hysteresis loop for the case of reduced anisotropy constant $\mu=-0.1$ and exchange parameter $\lambda$ $=0.05$.

Permalloy bilayer. They found this shift to be equal to the algebraic sum of the uniaxial anisotropy and the exchange bias fields, obtained with SQUID magnetometry. The relatively small difference $(\approx 20 \%)$ between the exchange bias field value determined by FMR and that obtained through hysteresis measurements by McMichael et al. ${ }^{16}$ for $\mathrm{Ni}_{80} \mathrm{Fe}_{20} / \mathrm{NiO}$ (attributed by the authors to asymmetric magnetization reversal mechanisms), also supports our conclusion.

The analytical expression for $\left|h_{e b}^{a c}\right|=J_{\mathrm{ac}}(\lambda) / \sigma_{w}$ has been obtained by Xi et al. assuming that (by analogy with another case of an exchange-biased system simpler than the one considered here) the loop shift is the first term of the denominator of the $\chi$ expression [Eq. (26)]. The denominator, however, represents the effective anisotropy field, and not the algebraic sum of the exchange bias field and the uniaxial anisotropy field (coercivity). One can see that in regions I, IV, and $\mathrm{V}$, the $h_{e b}$ does not depend on $\mu$ explicitly, but which of the $h_{e b}$ expressions should be used is determined by the inequalities involving $B_{1}, B_{2}, B_{3}$, and $B_{4}$, and all these expressions depend on $\mu$. Actually, none of the five expressions for $h_{e b}$ has form identical with the first term of the denominator of Eq. (26), even for the case of $\mu=0$. Thus the expression given by $\mathrm{Xi}$ et al. does not represent the exchange bias field for systems described by the Mauri's model and, consequently, cannot be compared with the ones obtained for other measurement techniques. The quantities which can be compared from easy axis hysteresis loop measurements and hard axis ac susceptibility measurements, are the field for which the descending part of a normalized hys- 
teresis loop starts to decrease from 1 (i.e., $h_{0,0}$ ), and the effective anisotropy field obtained from a hard axis ac susceptibility curve, respectively.

Although the experimentally measured hysteresis loops have not been shown in the paper of Ström et al. ${ }^{14}$ the difference between the exchange bias field values estimated by ac susceptibility and through hysteresis loop measurements for their $\mathrm{Co} / \mathrm{CoO}$ bilayers can be explained in light of the discussions above. In the energy expression of the very simple phenomenological model used in the work of Ström et al., only the terms corresponding to the exchange anisotropy and the magnetostatic energy have been considered, and $h_{e b}^{a c}$ was found to be the denominator of the $\chi$ expression. The uniaxial anisotropy, however, cannot be ignored for $\mathrm{Co} /$ CoO bilayers: for thin Co films it might be a little smaller than for bulk Co $\left(5 \times 10^{6} \mathrm{erg} / \mathrm{cm}^{3}\right)$, but exchange biased FM films always show an enhanced coercivity associated with the AF layer. ${ }^{12}$ Hence more complex model should be used to describe the behavior of $\mathrm{Co} / \mathrm{CoO}$ bilayers. As discussed above, in the framework of the model of Mauri et al., $M_{s} / \chi$ represents the effective anisotropy field and not $h_{e b}^{a c}$ (for $\chi$ obtained from hard axis measurements) and consequently, the measured $M_{s} / \chi$ values could be much higher than the $h_{e b}$ obtained from easy axis hysteresis loop data. This is demonstrated in Fig. 5, where the hard axis magnetization and susceptibility curves, as well as the easy axis hysteresis loop for the case of $\mu=-0.1$ and $\lambda=0.05$ are plotted. The corresponding values for $\chi_{\max }, M_{s} / \chi_{\max }, h_{0,0}$, and $h_{e b}$ are given as well. The $M_{s} / \chi_{\max }$ is five times higher than $h_{e b}$ in this particular case, and is equal to $h_{0,0}$, as expected.

\section{SUMMARY}

In the present work, analytical expressions were derived for the exchange bias field, coercivity and effective anisotropy field in FM/AF bilayers in the framework of the model of Mauri et al. It was demonstrated that, depending on the interfacial exchange coupling strength and the FM anisotropy, there are five different sets of analytical expressions for the hysteresis loop displacement and coercivity, and only one expression for the effective anisotropy field. These expressions were compared with the previously reported theoretical results, and their validity was discussed.

It was shown that, contrary to the $\mathrm{Xi}$ et al. conclusions, the hysteresis loop, ac susceptibility, and FMR measurements of exchange anisotropy for magnetic systems whose behavior can be described in the framework of the Mauri et al.'s model, should not give different values for the exchange bias field. The difference between the exchange bias field values, estimated experimentally by ac susceptibility and through hysteresis loop measurements for $\mathrm{Co} / \mathrm{CoO}$ bilayers, was explained as well.

\section{ACKNOWLEDGMENTS}

The author thanks Dr. S. M. Rezende for motivating his initial interest in exchange anisotropy and for critical reading of the manuscript, and to Dr. L. G. Pereira for the helpful discussions. This work has been supported by Conselho Nacional de Desenvolvimento Científico e Tecnológico (CNPq, Brazil), Fundação de Amparo à Pesquisa do Estado do Rio Grande do Sul (FAPERGS, Brazil), and Financiadora de Estudos e Projetos (FINEP, Brazil).

${ }^{1}$ W.H. Meiklejohn and C.P. Bean, Phys. Rev. 102, 1413 (1956); 105, 904 (1957).

${ }^{2}$ R.D. Hempstead, S. Krongelb, and D.A. Thompson, IEEE Trans. Magn. MAG-14, 521 (1978).

${ }^{3}$ D. Heim, R. Fontana, C. Tsang, V. Speriosu, B. Gurney, and M. Williams, IEEE Trans. Magn. MAG-30, 316 (1994).

${ }^{4}$ A.P. Malozemoff, Phys. Rev. B 35, 3679 (1987).

${ }^{5}$ D. Mauri, H.C. Siegmann, P.S. Bagus, and E. Kay, J. Appl. Phys. 62, 3047 (1987).

${ }^{6}$ N.C. Koon, Phys. Rev. Lett. 78, 4865 (1997).

${ }^{7}$ T.C. Schulthess and W.H. Butler, Phys. Rev. Lett. 20, 4516 (1998)

${ }^{8}$ B.H. Miller and E.D. Dahlberg, Appl. Phys. Lett. 69, 3932 (1996).

${ }^{9}$ M.D. Stiles and R.D. McMichael, Phys. Rev. B 59, 3722 (1999).

${ }^{10}$ H. Xi and R.M. White, Phys. Rev. B 61, 80 (2000).

${ }^{11}$ N.J. Gökemeijer, J.W. Cai, and C.L. Chien, Phys. Rev. B 60, 3033 (1999).

${ }^{12}$ J. Nogués and Ivan K. Schuller, J. Magn. Magn. Mater. 192, 203 (1999).

${ }^{13}$ A.E. Berkowitz and K. Takano, J. Magn. Magn. Mater. 200, 552 (1999).

${ }^{14}$ V. Ström, B.J. Jönsson, and D. Dahlberg, J. Appl. Phys. 81, 5003 (1997).

${ }^{15}$ P. Miltényi, M. Gruyters, G. Güntherodt, J. Nogués, and Ivan K. Schuller, Phys. Rev. B 59, 3333 (1999).

${ }^{16}$ R.D. McMichael, M.D. Stiles, P.J. Chen, and W.F. Egelhoff, Jr., Phys. Rev. B 58, 8605 (1998).

${ }^{17}$ H. Xi, R.M. White, and S.M. Rezende, Phys. Rev. B 60, 14837 (1999).

${ }^{18}$ J. Geshev, M. Mikhov, and J.E. Schmidt, J. Appl. Phys. 85, 7321 (1999).

${ }^{19}$ M. Cougo dos Santos, J. Geshev, J.E. Schmidt, S.R. Teixeira, and L.G. Pereira, Phys. Rev. B 61, 1311 (2000).

${ }^{20}$ M. Rubinstein, P. Lubitz, and Shu-Fan Cheng, J. Magn. Magn. Mater. 195, 299 (1999). 